Advances in Science, Technology and Engineering Systems Journal Vol. 3, No. 6, 438-445 (2018)

www.astesj.com

Special Issue on Recent Advances in Engineering Systems
ASTESJ

ISSN: 2415-6698

\title{
Study on CD ROADM Contention Blocking
}

\author{
Guangzhi $\mathrm{Li}^{*}, 1$, Kerong $\mathrm{Yan}^{2}$, Li Huang ${ }^{2}$, Bin $\mathrm{Xia}^{2}$, Fanhua Kong ${ }^{2}$, Yang $\mathrm{Li}^{2}$ \\ ${ }^{1}$ Futurewei Technologies, Inc., 400 crossing road, Bridgewater, NJ 08807, USA \\ ${ }^{2}$ Huawei Technologies Co., Ltd., Shenzhen, 518129, China
}

A R T I C L E I N F O

Article history:

Received: 18 October, 2018

Accepted: 05 December, 2018

Online: 19 December, 2018

Keywords:

CD ROADM

Contention Blocking

Contention Avoidance

\begin{abstract}
A B S T R A C T
Service providers are transferring their static optical transport networks from semipermanent connections to agile automatic switched optical networks (ASON) with dynamic optical connection provisioning and restoration. To achieve this goal, service providers are looking for flexible optical network ROADMs with CDC capabilities. Although many contention scenarios during network connection provisioning and restoration have been illustrated, surprisingly academic simulations have showed that the blocking probability improvement of CDC ROADM comparing with CD ROADM is not significant. This is good news for service providers since most deployed optical networks are only CD ROADM capable, instead of CDC ROADM capable. How to make use of existing CD ROADMs to achieve network automation becomes an urgent challenge. In this paper, we present two research results to attack this challenge: (1) first, we built an analytical mode to estimate the CD ROADM contention blocking probability and show that when a CD ROADM add/drop local direction capacity occupation ratio is low or moderate, the contention blocking probability is not significant. From this model, we estimate that one can use a CD add/drop local direction capacity occupation ratio up to $75 \%$ before installing another $C D$ ROADM add/drop local direction or installing a CDC add/drop local direction when available. Simulation results on real network topologies and traffic matrices verified our recommendation; (2) second, we observed that most deployed optical networks are usually providing $100 \mathrm{G}$ or $200 \mathrm{G}$ per wavelength while majority applications are still requesting much smaller bandwidths and service providers often provide OTN (Optical Transport Network) over ROADM architecture for transport services. Since OTN provides electronic switching capability, in this paper, we present a new algorithm and methodology to make use of both OTN switch and CD ROADM to avoid service contention without using CDC ROADM.
\end{abstract}

\section{Introduction}

Color-less,

Direction-less, and

Contention-less Reconfigurable Add/Drop Multiplex (CDC-ROADM) architectures have recently generated considerable interests among service providers and optical transport vendors [1 7]. There are many technical papers and industry white papers to describe the benefits and applications of CDC ROADM. Comparing with CD ROADMs (Colorless and Directionless only), CDC ROADMs are able to offer additional flexibility and simplicity for optical wavelength planning and operation, especially for dynamic wavelength traffic as well as wavelength dynamic restoration. However CDC ROAMD brings extra

"Guangzhi Li, Futurewei Technologies, Inc., 400 crossing road, Bridgewater, NJ 08807, USA, Guangzhi.li@huawei.com

www.astesj.com

https://dx.doi.org/10.25046/aj030652 components and complexity, which increases ROADM cost. In field network deployments, the majority deployed optical networks are still CD ROADM only. Then following questions come to our mind when service providers upgrade their optical ROADM networks: (1) where does the CD ROADM contention come from? (2) How much is CD ROADM contention blocking? (3) Can we use an analytical formula to estimate CD ROADM contention blocking? (4) Most importantly, what should we do when CD ROADM contention blocking occurs?

In this paper, we present our two research results to answer above questions: (1) first, we built an analytical mode to estimate the CD ROADM contention blocking probability and show that when a $\mathrm{CD}$ add/drop local direction capacity occupation ratio is low or moderate, the contention blocking probability is not 
significant [8]. From this model, we estimate that one can use a CD add/drop local direction capacity occupation ratio up to $75 \%$ before installing another $\mathrm{CD}$ add/drop local direction or installing a CDC add/drop local direction when available. Simulation results on real network topologies and traffic matrices verified our findings; (2) second, we observed that currently most optical networks are usually providing $100 \mathrm{G}$ or $200 \mathrm{G}$ per wavelength while majority applications are still requesting much smaller bandwidths and service providers often design OTN (Optical Transport Network) over ROADM architecture for transport services. Since OTN provides electronic switching capability, in this paper, we present a new algorithm and methodology to make use of both OTN switch and CD ROADM to avoid service contention without using CDC ROADM.

\section{ROADM Architecture Comparison}

Early stage of optical transport network is typical point-topoint wavelength division multiplex (WDM) system which consists of two terminals connected by a pair of fibers. Each terminal contains an optical wavelength multiplexer and demultiplexer, amplifier, and transponders that interface client signals. The number of inline amplifiers placed between the terminals depends on the length and quality of fiber. Each transponder re-transmits its incoming client signal onto a particular wavelength of the optical grid (also called a channel) and the optical multiplexer combines these signals at different wavelengths together and transmits the combined signal over a fiber to the de-multiplexer at the other end. The de-multiplexer decomposes the multiplexed signal back into original signals at their respective wavelengths, which are re-transmitted by each receiving transponder into its client signal.

When a point-to-point demand (also called connection) is transported over two WDM systems, two OTs are needed: the first OT converts the first WDM system wavelength into the common short-reach wavelength $(\lambda 0)$, and the second OT converts the $\lambda 0$ short reach wavelength into the second WDM system wavelength. However, when the two WDM systems are of the same technology from the same vendor, one regenerator can be used to replace the two back-to-back OTs, avoiding the conversion to the common short-reach wavelength, and thus reducing the component costs.

Later on, Reconfigurable Optical Add Drop Multiplexer (ROADM) technologies for optical transport network have been deployed due to their high capacity and capital savings. A ROADM network typically includes a set of multi-degree nodes connected via fibers to form a mesh topology. Traffic may be added or dropped, regenerated, or expressed through at ROADM nodes. Inside ROADM node, each network side WSS (wavelength selective switch) is called one line direction, each add/drop side WSS is called add/drop local direction. At the add/drop local direction, the classic ROADM was designed with fixed wavelength transponders and directed tributary for each line direction, and it was called colored and directed ROADM. Each transponder only is allowed to transmit signal on a fixed wavelength and to a fixed direction, see Figure 1 for an example. This ROADM design is acceptable for static optical connections. Network planners could plan the connections carefully and deploy them as planned for quite a long time. As network traffic grows and become more and more dynamic, service providers prefer to automate optical connection provisioning without manual intervention. In such an operation scenarios, this colored and directed ROADM architecture is no longer able to satisfy this requirement. For example, to reroute an existing wavelength to a different direction or to reuse an existing transponder for a different wavelength, all require new ROADM add/drop architecture, which was colorless and directionless ROADM, the so called CD ROADM.

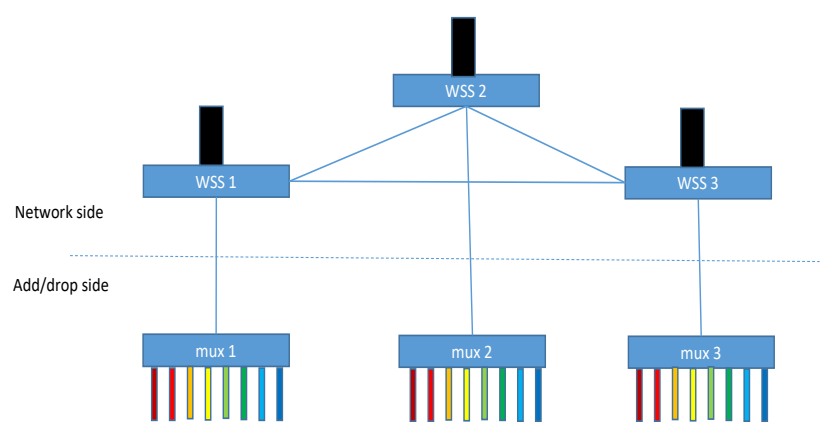

Figure 1: Example of colored and directed ROADM

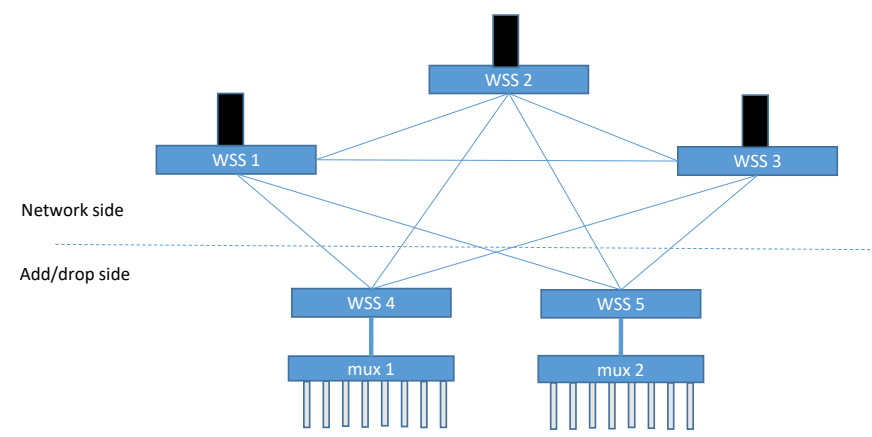

Figure 2: Example of CD ROADM

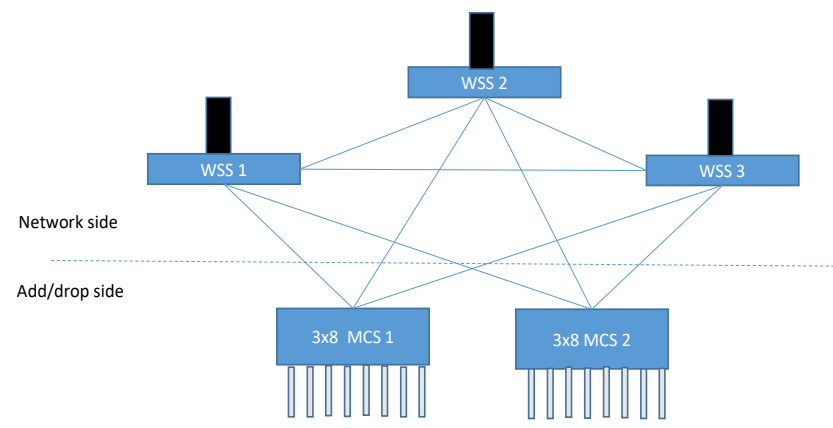

Figure 3: Example of CDC ROADM

Figure 2 shows an example of CD ROADM architecture with 3 line directions and 2 add/drop local directions. There are 8 tunable transponders, which means each transponder can be tuned to any wavelength. However, no more two transponders at the same add/drop local direction can be tuned to the same wavelength since all tuned wavelengths from transponders will be multiplexed into a single fiber pair. This limitation is called add/drop local direction contention. If two clients are connected to two transponders at a common add/drop local direction. Each client has a wavelength request at the same time, and routing wavelength assignment algorithm finds the same wavelength for the two requests. Due to add/drop local direction contention, the 


\section{G. Li et al. / Advances in Science, Technology and Engineering Systems Journal Vol. 3, No. 6, 438-445 (2018)}

two transponders could not use the same wavelength. Then either one of them has to find a different wavelength or may be blocked. This limitation of CD ROADM architecture leads to another advanced colorless directionless, contention-less ROADM architecture, the so called CDC ROADM.

Figure 3 shows an example of CDC ROADM architecture with 3 line directions and 2 local directions. In this architecture, the WSS (wavelength selective switch) and Multiplex are replaced by a $3 \times 8$ multicast optical switch (MCS). Without the fiber bottleneck of $\mathrm{CD}$ architecture, the transponders in same add/drop local direction can be tuned to the same wavelength, i.e., there is no wavelength contention in CDC ROADM architecture. This wavelength assignment flexibility is brought in by the relatively expensive component of MCS. In Figure 3, one can tune 3 transponders at a single add/drop local direction into a single wavelength since there are total 3 line directions. Of course, it is unnecessary to tune more than 3 transponders into a single wavelength since there are at most 3 common wavelengths can be supported at the network side. This extra flexibility should be able to improve network capacity utilization and reduce network blocking probability.

\section{CD ROAMD contention}

It is widely accepted that CD ROADM architecture causes wavelength contention during wavelength provisioning when two connections with the same wavelength need to be added/dropped at the same add/drop local direction [9 12]; during wavelength restoration when the network available wavelength has been used by other connection at the same add/drop local direction, and/or during regeneration when the connection wavelength is free at most one add/drop local direction only. To overcome CD ROADM contention issue, one may add as many local directions as the number of ROADM line directions. Then if there is one free wavelength at any line direction, there must exist one add/drop local direction with the same wavelength free. However during wavelength restoration, contention could still occur: during wavelength restoration, the transponder at the client side could be reused, and the restoration wavelength at network side is available while the restoration wavelength may not be available at add/drop local direction of the client transponder. To solve this contention, client-side optical cross connect architecture is recommended [10]. Problem seems solved, the issue is the extra cost of cross-connect. At each ROADM site, the number of connection requests usually much less than the value of $\mathrm{M}^{*} \mathrm{~W}$, where $\mathrm{M}$ is the number of line directions and $\mathrm{W}$ is the number of wavelengths per fiber. Large number of add/drop local directions will reduce the available number of potential line directions for the same size of WSS, which makes network expansion difficult. In real deployed optical networks, the number of add/drop local directions usually is much smaller than the number of line directions. In this case, an expensive CDC add/drop local direction could be used to avoid wavelength contention.

All above analysis is quantitative with extreme case assumptions. How severe is the CD ROADM contention during optical network planning and operation? Academic studies show that under reasonable assumption on network topologies and demands, the blocking probability improvement using CDC ROADM comparing CD ROADM with the same number of add/drop local directions at each node is not significant [13 15].
This surprising observations brought us interests for a further investigation at CD ROADM contention issue, including theoretical analysis and simulation verification, as well as how to resolve CD ROADM contention.
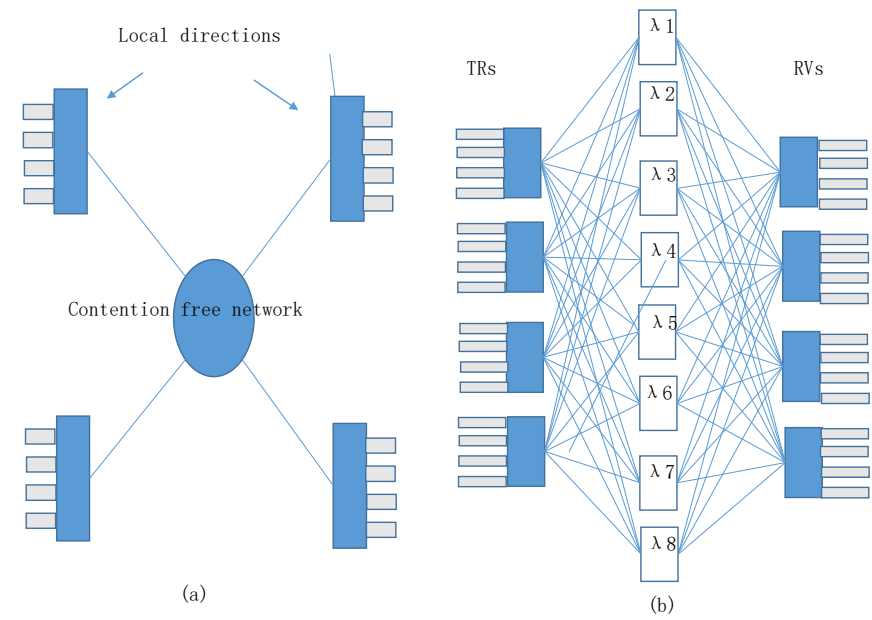

Figure 4: Clos model of CD ROADM

A connection in a CD ROADM network is blocked when the network could not find a free wavelength, or the wavelength is not available at either side of add/drop local directions. The first one is due to optical network wavelength continuity constraint and the second is due to $\mathrm{CD}$ wavelength contention. Without considering network wavelength continuity constraint, we could simply model the CD ROADM network as a star topology, see figure 4(a): the network is a full contention free optical switch at the center, each $\mathrm{CD}$ add/drop local direction is a star terminal. Assume there are $\mathrm{n}$ tunable transponders at each terminal, and each star branch fiber supports $\mathrm{m}$ wavelengths. Each transponder can be tuned to any wavelength, but no more two transponders can be tuned to a same wavelength. Then two terminals are able to establish a connection if and only if the two terminals have two transponders and the two branch fibers have a common free wavelength. If there are total $r$ add/drop local directions, the simplified star model can be viewed as a $(r, n, m)$ clos network [16], see figure 4(b), where a single transponder is separated into one transmitter (TR) and one receiver (RV). Thus the non-blocking condition would be $m \geq 2 n$ 1 , i.e., $\mathrm{n} \leq(\mathrm{m}+1) / 2$. So when an add/drop local direction is half filled, in order to avoid contention, it would be the time to add new $\mathrm{CD}$ add/drop local directions, or upgrade CDC add/drop local directions. Of course this simplified model has drawbacks since the optical network is not contention free at all. Even if two add/drop local directions have free transponders and same available wavelengths, but the network may not have the same wavelength between the associated two nodes; on the other hand, if the associated two node has an available wavelength along a path between them, the two add/drop local directions may not have the same common wavelength available. Thus our model is under estimate the contention blocking. However since there could be large number of paths between any two network nodes in real networks, we think this estimation may not be too off from reality. In next sections, we will verify our simplified model via simulation comparing CD ROADM and CDC ROADM of real networks with real demand matrix.

Academic papers show that even if the $\mathrm{CD}$ add/drop local direction traffic is more than half-filled, the blocking probability 
is still relatively low. When we want to create a connection between two $\mathrm{CD}$ add/drop local directions, again we look at the clos network model, which means we want to establish a connection from one TR bank $I$ of the first stage to one RV bank $J$ of the third stage. We define clos network state $\{u, v\}$ of TR bank $I$ and RV bank $J$ as $u$ TRs are busy and $v$ RVs are also busy, and define $B(u, v)$ as the contention blocking probability in state $\{u, v\}$ independent of other TR banks and RV banks.

According to Jacobaeus [17], the contention blocking probability $B(u, v)$ in state $\{u, v\}$ is:

$$
B(u, v)= \begin{cases}0 & \text { when } u+v<m \\ \frac{u ! v !}{m !(2 n-m) !} \text { when } u+v \geq m\end{cases}
$$

Simple proof: when $u+v<m$, there is at least one wavelength available in the second stage, which is reachable for both TR bank $I$ and RV bank $J$, so no blocking for a new connection. When $u+v$ $\geq m$, depending on how $u$ and $v$ connect to the second stage wavelengths, there could be no more wavelength available for next connection if and only if RV bank $J$ all available wavelengths $(m-v)$ resides in TR bank $I$ busy wavelengths. So the blocking probability could calculated as:

$$
\begin{aligned}
& B(u, v) \\
& =\frac{\text { choosing } m-v \text { wavelengths from } u \text { wavelengths }}{\text { choosing } m-v \text { wavelengths from } m \text { wavelengths }} \\
& =\left(\begin{array}{c}
m-v \\
u
\end{array}\right) /\left(\begin{array}{c}
m-v \\
m
\end{array}\right)=\frac{u ! v !}{m !(u+v-m)} .
\end{aligned}
$$

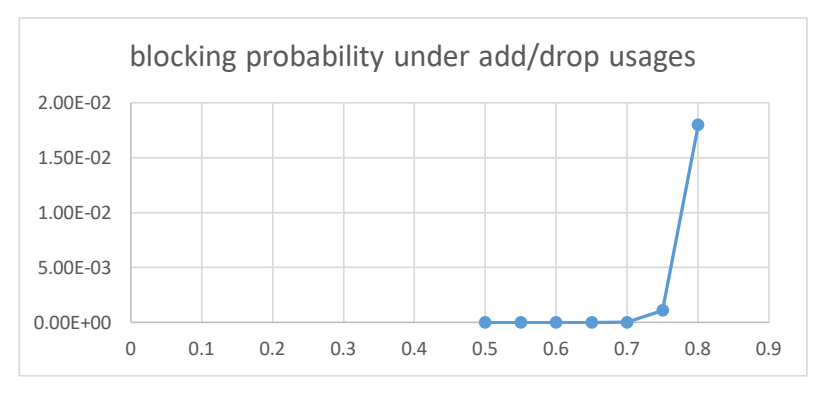

Figure 5: CD ROADM estimated blocking probability

Now we let $u=v=x * m$, where $m=80$, and calculate the blocking probability of $x=0.5,0.55,0.6,0.65,0.7,0.75,0.8$. See Figure 5, where $\mathrm{x}$-axis is the value of $x$ for number of existing connections in $\mathrm{CD}$ add/drop local directions, and y-axis is the estimated blocking probability. It is easy to see that even if the add/drop local direction is 80 percent full, the contention blocking probability is still under $2 \%$ without considering network wavelength continuity constraint. When the $\mathrm{CD}$ add/drop dimension is $75 \%$ full or less, the contention blocking probability is still negligible. Based on this analytical modeling, it explains why all academic simulations reported very small blocking probability using CD ROADM architecture. In next sections, we will use real network topology and network traffic matrix to simulate CD ROADM blocking probability. Our results confirm this analysis with very small variance.

\section{An example interpretation of clos model}

In section 3, we model CD ROADM as a clos network model and claim that when the CD ROADM local directions are not half filled, the CD ROADM blocking probability will be similar to CDC ROADM blocking probability. This claim may not be easy to interpret. In this section, we give one simple example to show that when CD ROADM local direction is half-filled, some CD ROADM connections will be blocked while the same network configuration with CDC ROADM will not block those connections.

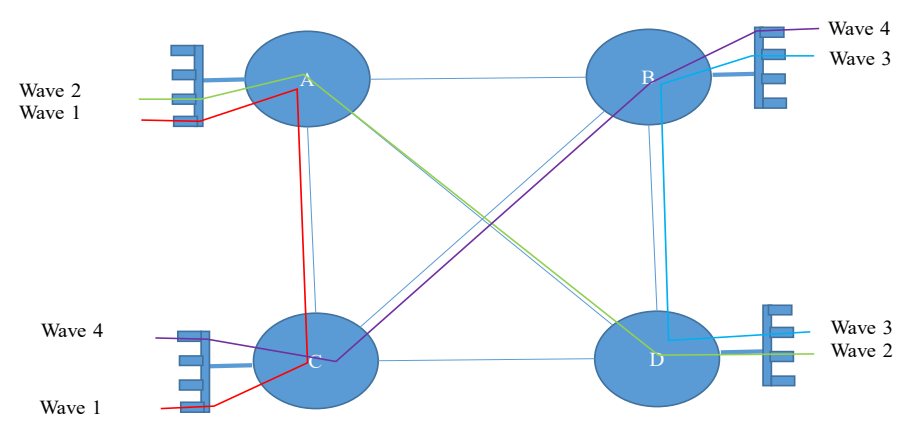

Figure 6: Example of CD ROADM half-filled blocking

Figure 6 shows a 4 node ROADM network and each node is deployed with a single $\mathrm{CD}$ add/drop local direction. Without loss of generosity, we assume that each fiber supports 4 wavelengths. Due to network dynamic operation and wavelength connections arrive and leave. At some network stage, we assume there are only 4 connections: connection 1 from node $\mathrm{A}$ to node $\mathrm{C}$ with wavelength 1 ; connection 2 from node $\mathrm{A}$ to node $\mathrm{D}$ with wavelength 2 ; connection 3 from node $\mathrm{B}$ to node $\mathrm{D}$ with wavelength 3 ; and connection 4 from node $\mathrm{B}$ to node $\mathrm{C}$ with wavelength 4 . If there is a new connection request from node $\mathrm{A}$ to node $\mathrm{B}$, the request will be blocked due to CD ROADM local contention, while $\mathrm{CDC}$ architecture will be able to provision such connection request; similarly another connection request from node $\mathrm{C}$ to node $\mathrm{D}$ will be blocked also in $\mathrm{CD}$ architecture while CDC architecture will not block it. In this simple example, it is easy to see that CD ROADM local direction half-filled is a critical point to cause contention blocking.

\section{CD/CDC ROADM network simulation}

We first simulate a few planed or planning CD ROADM optical networks under preplanned demand matrixes with and without rerouting restoration. Table 1 shows the parameters of these networks, where \#degree is calculated as $2 *(\# l i n k s) /(\#$ nodes $)$, where \#links means the number of network links, and \#nodes means the number of network nodes. The \#add/drop direction distribution $X(Y)$ means $y$ network nodes with $x$ add/drop local directions. We first assume all add/drop local directions are $\mathrm{CD}$ architecture, then using a multipath routing and first-fit wavelength assignment algorithm to provision the connections as many as possible. 
G. Li et al. / Advances in Science, Technology and Engineering Systems Journal Vol. 3, No. 6, 438-445 (2018)

Table 1: Simulated network parameters

\begin{tabular}{|l|l|l|l|l|l|}
\hline networks & \#node & \#link & \#degree & \#connections & \#add/drop direction distribution X(Y) \\
\hline Net 1 & 30 & 71 & 4.73 & 324 & $2(2), 1(18), 0(10)$ \\
\hline Net 2 & 28 & 43 & 3.07 & 404 & $2(5), 1(23)$ \\
\hline Net 3 & 55 & 81 & 2.95 & 723 & $3(2), 2(4), 1(47)$ \\
\hline
\end{tabular}

Table 2: Simulation results of CD vs CDC

\begin{tabular}{|l|l|l|l|l|l|l|l|l|}
\hline networks & $\begin{array}{l}\text { CD (W) } \\
\text { blocking }\end{array}$ & $\begin{array}{l}\text { CDC }(\mathrm{W}) \\
\text { blocking }\end{array}$ & $\mathrm{R}(\mathrm{CD})$ & $\mathrm{R}(\mathrm{CDC})$ & $\begin{array}{l}\text { Extra CD for } \\
\text { working }\end{array}$ & $\begin{array}{l}\text { Extra CD for } \\
\text { rerouting }\end{array}$ & $\begin{array}{l}\text { Max CD fill ratio } \\
\text { before extra CD }\end{array}$ & $\begin{array}{l}\text { Max CD fill ratio } \\
\text { after extra CD }\end{array}$ \\
\hline Net 1 & 0 & 0 & $0.43 \%$ & 0 & 0 & 8 & $86.25 \%$ & $43.50 \%$ \\
\hline Net 2 & $7.4 \%$ & $1.7 \%$ & $16.5 \%$ & $16.9 \%$ & 3 & 3 & $85 \%$ & $56.70 \%$ \\
\hline Net 3 & $3.87 \%$ & $1.93 \%$ & $6 \%$ & $3.75 \%$ & 12 & 12 & $88.80 \%$ & $53.00 \%$ \\
\hline
\end{tabular}

For those provisioned connections, we simulate single failure rerouting and calculate the maximal success rerouting ratio:

$R(C D)=\frac{\text { total rerouting sucess connections over all failures }}{\text { total failed connections over all failures }}$

Then we assume all add/drop local directions are CDC architecture, then using the same algorithm to provision the connections as many as possible. For all provisioned connections, we also simulate single failure rerouting and calculate the maximal success rerouting ratio, $\mathrm{R}(\mathrm{CDC})$.

To investigate the CD contention impact, we try to add extra $\mathrm{CD}$ add/drop local directions to critical nodes such that the CD blocked demands equal to $\mathrm{CDC}$ blocked demands. The CD add/drop local direction adding policy is based on decreasing ratio of (\#add/drop_connections)/(80*\#add/drop_direction) at each network node. We simulated both with and without failure rerouting cases. Table 2 shows our simulation results, where "Extra CD for working" means total extra CD local directions for working only with the same blocking ratio as CDC ROADM architecture, "Extra CD for rerouting" means total extra CD local directions for working and restoration with the same blocking ratio as $\mathrm{CDC}$ architecture, "Max $\mathrm{CD}$ fill ratio before extra CD" means maximal $\mathrm{CD}$ add/drop local direction fill ratio before adding extra $\mathrm{CD}$ add/drop local directions, and "Max $\mathrm{CD}$ fill ratio after extra CD" means maximal CD add/drop local direction fill ratio after adding extra $\mathrm{CD}$ add/drop local direction for rerouting. We observed that for all tested real networks, the differences between $\mathrm{CD}$ and $\mathrm{CDC}$ are relatively small. Especially when node CD fill ratio (total \#add/drop demands divided by link capacity times \#add/drop directions) is less than 50\%, CD and CDC outputs are almost the same, which means that CDC does not provide any extra benefits in this case; on the other hand, under the same network topology and demand matrix, in order to achieve the same throughput and rerouting success ratio, CDC did require relatively fewer add/drop local directions, but not significant.

All these tests are based on off-line planning algorithms with static traffic demands. In order to simulate dynamic traffic, we also created a hypothetic network with 50 nodes, 123 links with average node line directions about 4 . Link capacity is 80 , and each node has one add/drop local direction. In this simulation, we assume paths between any two nodes are all reachable and no regenerator is used. Demands are random generated with poison arrival and expectation holding time. We measure the Erlang $\underline{\text { www.astesj.com }}$ value when blocking probability is under $1 \%$. We observed that $\mathrm{CDC}$ architecture could improve the throughput of Erlangs about $4 \%$ comparing with CD architecture, see Figure 7. Again CDC is able to improve dynamic traffic throughput, but the improvement is not significant.

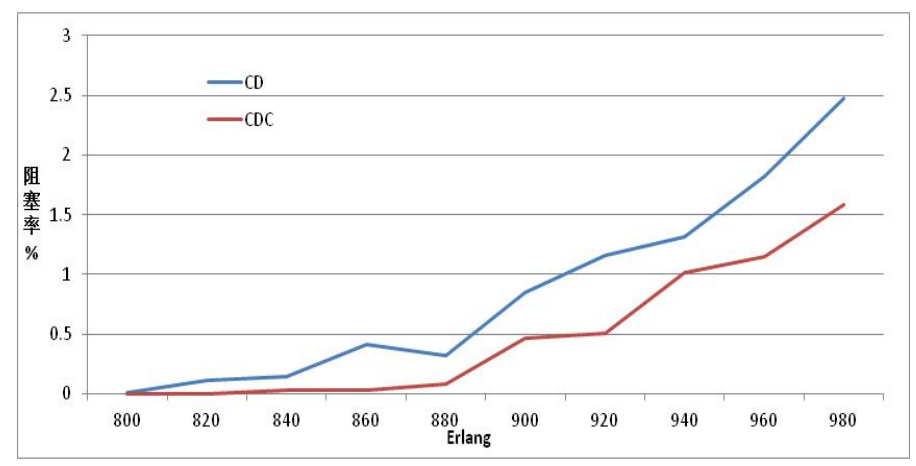

Figure 7: Throughput improvement of CDC vs CD

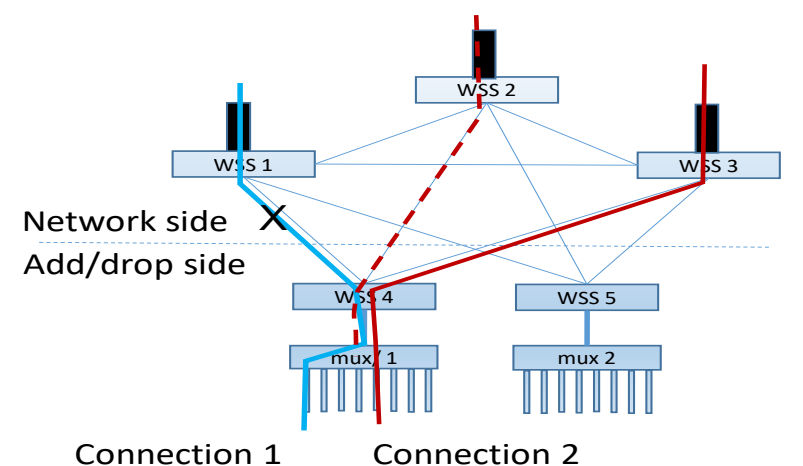

Figure 8: Example of CD ROADM contention

\section{A Contention Solution for CD ROADM}

Although CD ROADM contention blocking is small when add/drop local direction utilization is low or medium, there is still some possibility of contention blocking during dynamic optical network operation, such as ASON (automatic switch optical network) connection provisioning or restoration. For example in Figure 8, assuming local direction WSS 4 has two connections of 1 and 2 , connection 1 was assigned wavelength 1 and routed along WSS 1 line direction while connection 2 was assigned wavelength 2 and routed along WSS 3 line direction. If connection 1 fails and required rerouting restoration, and the source node finds out that 


\section{G. Li et al. / Advances in Science, Technology and Engineering Systems Journal Vol. 3, No. 6, 438-445 (2018)}

only wavelength 2 along WSS 2 line direction is available and other wavelengths along other WSS line directions are not available. But due to add/drop local direction WSS 4 wavelength contention, wavelength 2 could not be used for connection 1 rerouting, which could lead to connection 1 rerouting failure. If this ROADM is CDC architecture, connection 1 is able to be routed to WSS 2 line direction with free wavelength 2 .

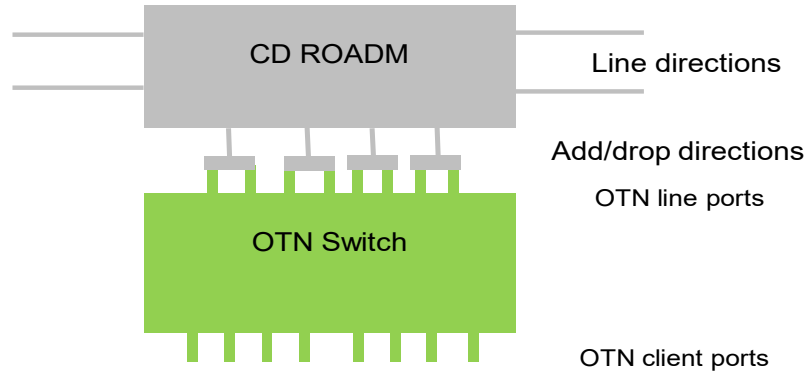

Figure 9: OTN over ROADM architecture

As we mentioned before, CDC ROADM relies on MCS module to multicast each wavelength to each direction, which increases hardware cost and system complexity. In this paper, we present a new solution to avoid CD ROADM wavelength contention during wavelength provisioning and rerouting using existing mature technology without increasing hardware cost. We noticed that most of ROADM node deployments are armed with OTN electronic switching node to form an OTN over ROADM architecture, see Figure 9, where OTN switch is used for traffic grooming and ROADM is used for wavelength switching. However such OTN over CD ROADM architecture still could not be equivalent to CDC ROADM architecture. For example, assume a CD ROADM has 4 line directions, 4 add/drop local directions, and each fiber has 4 wavelengths. Since OTN line ports are pre-installation in field operation, assuming 50\% connections add/drop per node, the service provider pre-installed 8 OTN line ports and their usage is based on average policy as recommended by paper [10]. If connection wavelength sequence order is $1,1,1,2,3,3,3,2$, the last connection would be blocked due to wavelength contention, however CDC ROADM won't have this contention. However under OTN over ROADM architecture, we have following observations:

Observation 1: wavelength contention blocking condition is that for a given wavelength, all add/drop local directions with free OTN line ports have used the given wavelength.

Observation 2: if CD ROADM has equal number of add/drop local directions as line directions, and one line direction has a free wavelength, then at least one add/drop local direction has the same free wavelength.

Observation 3: given a connection routing wavelength, if the add/drop local direction with the free given wavelength has free OTN line ports connected, the connection can be deployed without contention;

Observation 4: given a connection routing wavelength, assuming equal number of add/drop local directions and line directions, then according to observation 2 , at least one add/drop local direction has the wavelength free, say WSS X, and X has no free OTN line port, but add/drop local direction WSS Y has free OTN line port. If OTN line ports are equally connected to CD ROADM add/drop local directions, then WSS X at most has one OTN line port less than WSS Y. So WSS X at least has one wavelength occupied but WSS Y is still free. This is because if WSS Y occupied all wavelengths of WSS X used, and WSS Y also used the given new wavelength, and free OTN line port, then WSS Y would have at least 2 more OTN line ports than WSS X, which contradicts the assumption of OTN line ports equal distribution to ROADM local directions.

Observation 5: if we can change the free OTN line port (say y) of WSS Y into the wavelength that WSS X used but WSS Y is free, say $\lambda 1$, identify wavelength $\lambda 1^{\prime}$ ROADM line direction (say d), cross-connect the OTN line port y to ROADM line direction $d$, switch the service OTN client port to OTN line port y, free WSS $X$ wavelength $\lambda 1$ occupied OTN line port (say $z$ ), then WSS X has free wavelength $\lambda$, and connection free ONT line port $z$, thus the new connection can be provisioned without contention.

Observation 6: to achieve hitless switching, one could use makebefore-break technology; however traditional make-before-break usually is used on the same layer of technology, such as in optical layer or electronic layer, in this proposal, the make-before-break is used in cross-layer between electronic layer and optical layer. We have discussed with both optical system engineers and electronic system engineers and was confirmed that it can be done using existing technologies.

\section{Two Use Cases}

In this section, we provide two use cases on how to apply the method of section 6 to avoid CD ROADM contention.

\subsection{Use case 1: service provisioning contention blocking avoidance}

Figure 10 shows an OTN over CD ROADM service provisioning contention blocking avoidance process. Assume the OTN, has 8 client ports numbered from 1 to 8 , and 8 line ports connecting to $4 \mathrm{add} / \mathrm{drop}$ local directions of a CD ROADM, numbered as $\mathrm{x} . \mathrm{y}$, where $\mathrm{x}$ is the CD ROADM add/drop local direction number, $y$ is the line port sequential order number at the local direction. For example 2.1 means the first line port at the second local direction of the ROADM.

We further assume that each fiber supports 4 wavelengths, OTN client port 1 cross-connects to line port 1.1 with wavelength 1 , client port 2 cross-connects line port 2.1 with wavelength 1 , client port 3 cross-connects line port 3.1 with wavelength 2 , client port 4 cross-connects to line port 4.1 with wavelength 2 , client port 5 cross-connects to line port 1.2 with wavelength 3 , client port 6 cross-connects line port 2.2 with wavelength 3 , client port 7 crossconnects to line port 3.3 with wavelength 3 . At this time, there is still one free client port 8 and one free line port 4.2. If a new service finds available wavelength 2 over one line direction, the service cannot be deployed since CD ROADM local direction 4 already occupied wavelength 2 (see figure 10.a). Contention blocking occurs and following procedure could resolve this contention.

$\checkmark \quad$ Identify free line port: 4.2 on local direction 4

$\checkmark \quad$ Identify one local direction with free wavelength 2: local direction 1 .

$\checkmark$ Identify one OTN line port with occupied wavelength from local direction 1 which is free at local direction 4: line port 


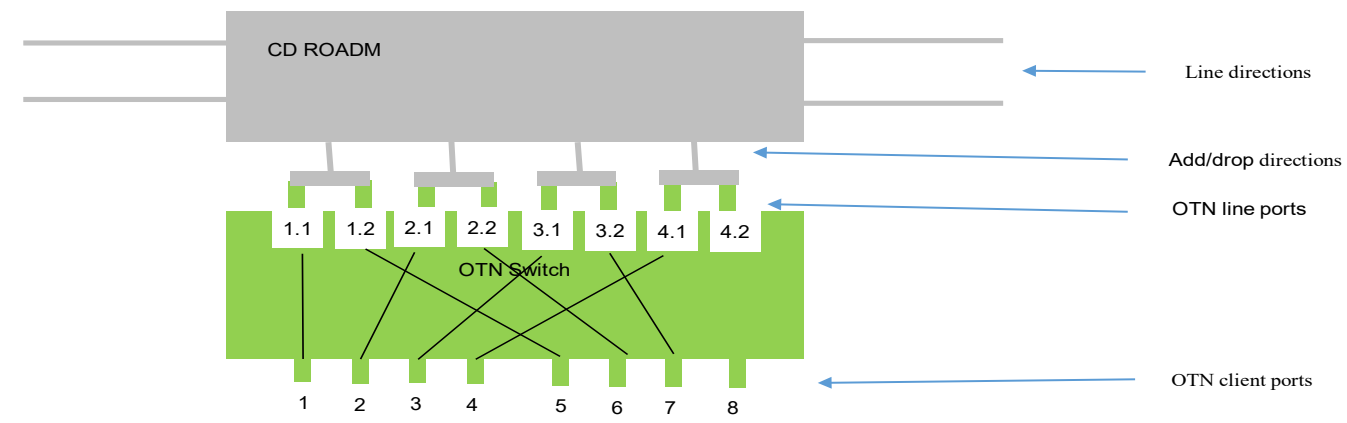

(a)

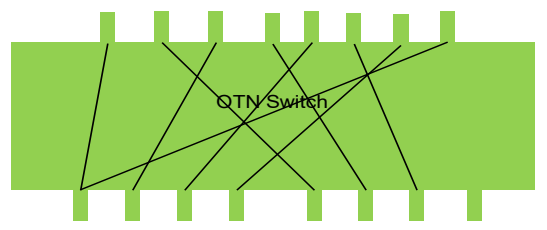

(b)

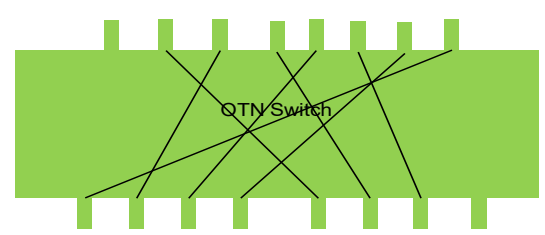

(c)

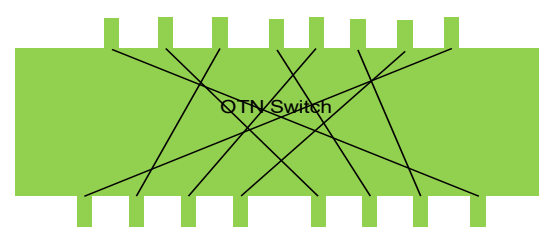

(d)

Figure 10: OTN over ROADM provisioning contention avoidance

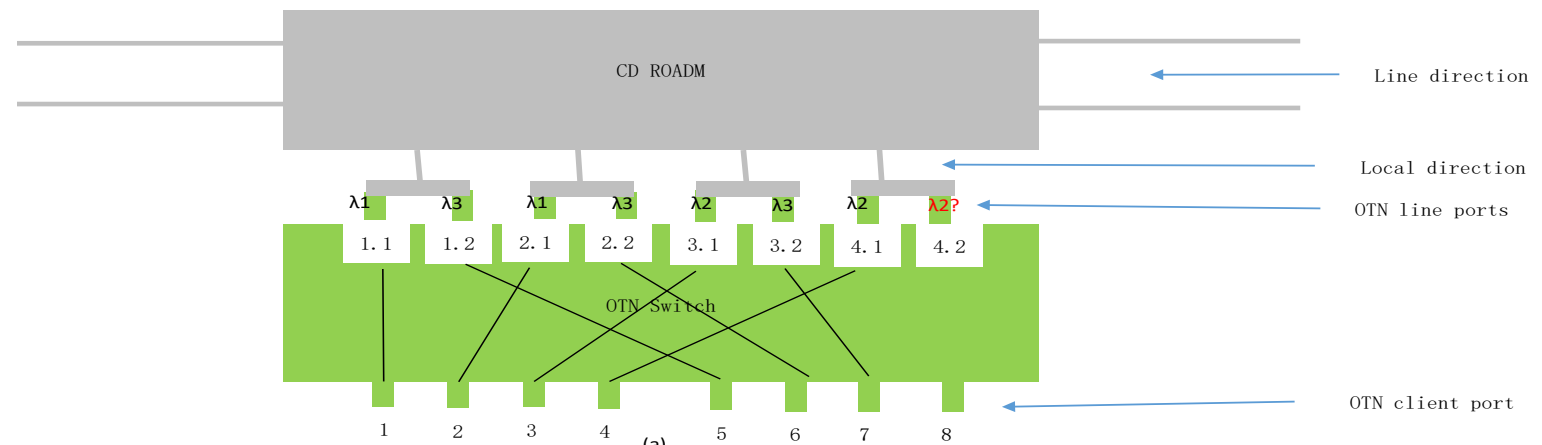

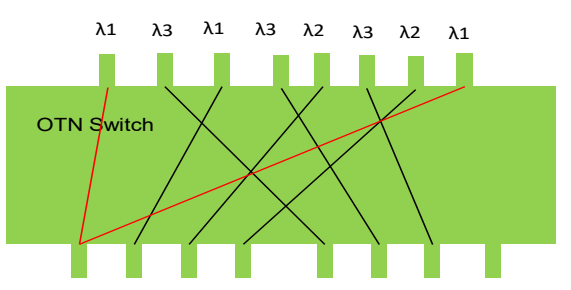

(b)

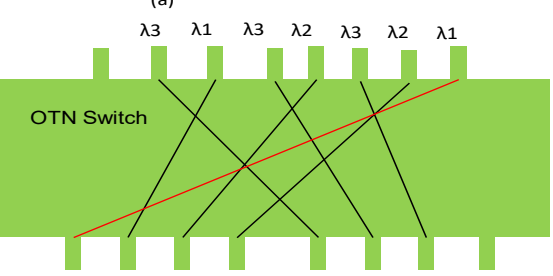

(c)

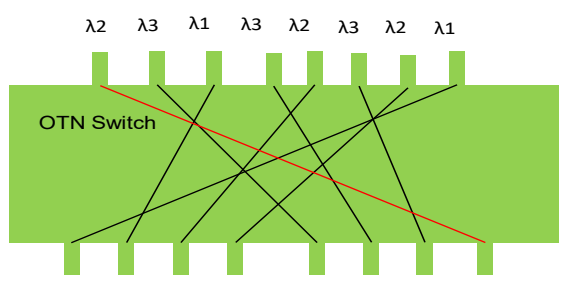

(d)

Figure 11: OTN over ROADM rerouting contention avoidance

1.1 with wavelength 1 . This can be done according to section 6 observation 4

$\checkmark \quad$ Identify line port 1.1 associated client port: client port 1 .

$\checkmark \quad$ Client port dual-cast to line ports 1.1 and 4.2, see figure 10.b

$\checkmark$ CD-ROADM cross-connects 4.2 signal to the same ROADM line direction of line port 1.1

$\checkmark$ ROADM line WSS of line port 1.1 selects signal from line port 4.2

$\checkmark \quad$ Client port cancels signal to line port 1.1, then line port 1.1 is free, see figure 10.c

$\checkmark$ Client port 8 new service cross-connects to line port 1.1 with wavelength 2 . See figure 10.d

\subsection{Use case 2: failure rerouting contention blocking avoidance}

Figure 11 shows the use case of service failure rerouting contention avoidance solution. Figure 11.a shows service OTN www.astesj.com internal configuration and wavelength assignment. When a service fails, wavelength rerouting restoration is activated. If rerouting wavelength is the same as original working service wavelength, then OTN configuration has no change, the only change is CD ROADM to cross-connect line port signal from original working line direction to rerouting line direction; If rerouting wavelength is different from original working wavelength, and the local direction of working wavelength has free rerouting wavelength, then the OTN configuration does not need to change, the only change is to tune the working line port to rerouting wavelength and ROADM to cross-connect the rerouting wavelength to rerouting line direction. However if rerouting wavelength is different from working wavelength, and the local direction of working wavelength has occupied the rerouting 
wavelength, then we need to find out all local directions with free rerouting wavelength. If one of them has free line port, then we could tune this line port to the rerouting wavelength, ROADM cross-connect the rerouting wavelength to rerouting line direction, and cross-connects the service client port to the rerouting wavelength line port. The difficult case is when all local directions with free rerouting wavelength have no free line ports, we need to adjust at most one existing service configuration. Assume OTN configuration as in figure 11 (a) and client port 7 service fails and rerouting wavelength is wavelength 2 . Then working wavelength local direction 3 has no free wavelength 2. After identifying all local directions having free wavelength 2 , we find local direction 1 and local direction 2. However both local directions have no free line ports. We choose one local direction with free rerouting wavelength and this local direction at least has one occupied wavelength which is not used by the local direction of failed working wavelength, this can be done according to section 6 observation 4. In Figure 11.a, we choose local direction 2, where wavelength 3 is not used by local direction 3 (failed working wavelength is not used anymore). We reuse OTN line port 3.2, which is free currently and tune it into wavelength 3 , duel-cast client port 6 service to both line port 2.2 and line port 3.2, (figure 11.b) cross-connect line port 3.2 signal to ROADM line direction of line port 2.2 connected line direction, and let this line direction WSS selects signal from line port 3.2, cancel signal cast from client port 6 to line port 2.2 and free line port 2.2 (figure 11.c), at last tune line port 2.2 to rerouting wavelength 2 and cross-connects client port 7 signal to line port 2.2, ROADM cross-connects line port 2.2 signal to rerouting line direction (figure 11.d)

\section{Conclusion}

In this paper, we investigated CD ROADM contention blocking problem. Since ROADM network operation is moving from static to dynamic, from $1+1$ failure protection to dynamic rerouting restoration, CDC ROADM architecture has attracted lots of attention. However most deployed ROADM networks are still CD architecture. Is CD ROADM a show stop for dynamic operation? How much contention blocking will CD ROADM produce? If CD ROADM contention happens, is there any solution to solve this contention? We have answered all above questions using analytical model, simulation, and configuration method of procedures. From mathematic model and simulation results, we showed that CD ROADM did produce some contention during network operation, but the contention blocking is not as significant as we originally thought. If the service fill ratio at each local direction is $50 \%$ or less, the contention blocking is almost negligible. When contention did occurs during network operation, if service providers are using OTN as grooming and ROADM as wavelength transmission, as the often used architecture in field deployments, we provided a method to reconfigure at most one existing service to avoid the contention blocking. Based on our study, we concluded that a service provider with CD ROADM optical network can move forward to ASON (automatic switched optical network) dynamic operation without worrying too much of CD ROADM contention blocking.

\section{References}

[1] Mina Paik, "4 Things we learned at \#OFC17", March 30, 2017, http://www.ciena.com/insights/articles/4-Things-We-Learned-atOFC17.html

[2] Fujitsu Network Communications Inc., "CDC ROADM applications and cost comparison", whitepaper, 2014 https://www.ofcconference.org/getattachment/188d14da-88ba-4a63-91d61 cc14b335d8b/CDC-ROADM-Applications-and-Cost-Comparison.aspx

[3] Nokia, "Benefits of CDC-F ROADMs", whitepaper, 2017, https://knect365.com/ngon/article/e081 ea00-9bc9-433d-ae2194617299be41/whitepaper-benefits-of-cdc-f-roadms

[4] Huawei Technologies Co., Ltd., "white paper on technological developments of optical networks", whitepaper, 2016, http://www-file.huawei.com//media/CORPORATE/PDF/white\%20paper/White-Paper-on-

Technological-Developments-of-Optical-Networks.pdf

[5] Sterling Perrin, "Building a fully flexible optical layer with next-generation ROADMs", heavy reading whitepaper, 2011, http://www-file.huawei.com//media/CORPORATE/PDF/white\%20paper/White-Paper-on-

Technological-Developments-of-Optical-Networks.pdf

[6] B. C. Collings,, "Advanced ROADM technologies and architectures," Los Angeles, CA, Paper Tu3D.3, OFC 2015.

[7] S. Poole, S. Frisken, M. Roelens, and C. Cameron, "Bandwidth-flexible ROADMs as network elements," Los Angeles, CA, Paper OTuE1, OFC 2011

[8] Guangzhi Li, Kerong Yan, Li Huang, Bin Xia, Fanhua Kong, Yang Li, "How much is CD ROADM contention blocking?", San Diego, CA, OFC 2018.

[9] L.Zong, H. Zhao, Z. Feng, and S. Chao, "Demonstration of ultra-compact contentionless Roadm based on flexible wavelength router," ECOC 2014.

[10] M. Feuer, S. Woodward, P. Palacharla, X. Wang, I. Kim, and D. Bihon, "Intra-node contention in dynamic photonic networks", JOLT, 29(4), Feb. 2011.

[11] Y. Li, L. Gao, G. Shen, and L. Peng, "Impact of ROADM colorless, directionless, and contentionless (CDC) features on optical network performance," JOCN, 4(11), Nov. 2012.

[12] J. Simmons, "a Closer look at ROADM contention," IEEE Comm. Mag, 55(2), pp 160-166, Feb 2017.

[13] F. Naruse, Y. Yamada, H. Hasegawa, and K. Sato, "Evaluations of OXC hardware scale and network resource requirements of different optical path add/drop ratio restriction schemes," J. Opt. Commun. and Netw., 4(11), pp B26-B34, Nov. 2012.

[14] T. Zami, P. Jenneve, and H. Bissessur, "Fair comparison of the contentionless property in OXC," Asia Commun. and Photonics Conf., Hong Kong, AM3G.3, 2015.

[15] J. Pedro and S. Pato, "Impact of add/drop port utilization flexibility in DWDM networks," JOCN, 4(11), Nov 2012.

[16] C. Clos, "A study of non-blocking switching networks," BSTJ, 32(2), pp 406-424, March 1953.

[17] C. Rigault, "Clos networks: a correction of the Jacobaeus result," Analysis of telecommunications, 57(11-12), pp 1244-1252, Nov 2002. 